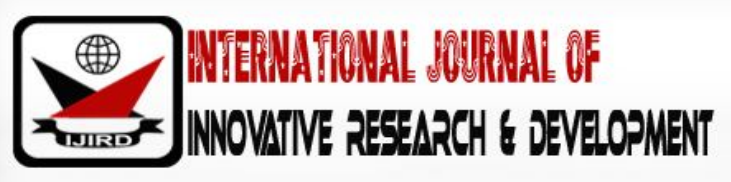

ISSN 2278 - 0211 (Online)

\section{The Place of Model Making in the Architectural Design Process: A Literature Review}

\author{
Fadeyi, Ayomipo Akintunde \\ Lecturer, Department of Architecture, Bells University of Technology, Nigeria \\ Adejokun, Paul Adeyinka \\ Student, School of Architecture, The University of Illinois at Chicago, USA \\ Abass, Dare Abideen \\ Student, Department of Architecture, Bells University of Technology, Nigeria
}

\begin{abstract}
:
Physical Model Making did not begin with the technological revolution that gave us hi-tech materials and the ability to build accurate models. Rather, it has a rich tradition dating back through the ages. Where there has been Architecture there has always been Physical Models. As a part of the design process, models provide a fairly quick mean to generate, communicate or get feedbacks on new ideas. At the turn of the twentieth century came the advent of Computer Aided Design (CAD) through which Virtual Models are being built. CAD is touted with so many advantages as a design tool. Can virtual model be substituted for physical models? When compared with physical models, it lacks feel and it remains trapped behind a twodimensional computer screen. Which makes the exploration of crucial ideas and presentation of others with physical models inevitable still date. The fact that physical models have survived all these years is an indication of how critical it is to the design process. Though the art have advanced with technology, the concept of it visualisation and it benefits of threedimensional thinking has remained the same. This paper reviews relevant literatures to account for the history of the art and how it became a part of architecture. Not dwelling on the methods, rather looking at the art and how critical it is as a "design tool". Furthermore, it impact on architectural designs was highlighted and evaluated in order to reach a conclusion on the place of model making in the architectural design process and what this portends for it future in architecture.
\end{abstract}

Keywords: Physical model, model making, architectural model, model history, design tool

\section{Introduction}

A model is the physical representation of a concept, whether realistic or abstract, with the intention of modifying, viewing, testing, and conveying its design (Phev, 2009). Through physical model making, designers explore their conceptual ideas and develop the creation and understanding of space (Salama \& Wilkinson, 2007). Buildings are three-dimensional in nature, hence representing it with a scale model makes sense the most, because when compared to other forms of representation, it is the closest to reality (Moon, 2005). It is a miniature of the real thing, showing what the finished product may look like and ensure that it can be built. Hence, if a picture is what a thousand words, it is not out of place to state that a model is worth multiple of presentation drawing sheets. The fundamental assumption for this statement is collaborated by Megan Werner, in her book Model Making, where she explains that the two-dimensional drawing leaves room for subjective interpretation whilst the three-dimensional scaled model is complete in its representation as it allows one to observe, analyze and project - it is objective (Werner, 2010). Kanya Phev also aligns with her opinion stating that with the use of a model, it is easy to see the design holistically without having to flip through a stack of different images (Phev, 2009).

Today's experience in architecture depicts the usage of sophisticated computer modelling and photorealistic renderings in architectural schools and practices. Though the reason why designers still use physical models in today's digital age remains unclear (Lei et al., 2014), physical models still features as an important part of presentations with students and the clients. Whether realistic or abstract, they are powerful communication component .Giving others the chance to view, discuss, and edit the design. Allowing different person sees something completely different, making them more engaged and open up discussions (Moon, 2005). From various pitches and presentations, in spite of the highly polished renderings presented, the clients and jury will always gravitate towards the model, no matter how crude, because physical models tends to enable quicker and more accurate comparisons of building forms (Lei et al., 2014). A proof that models have a tangibility that other presentation methods do not have. Which gives models it relevance in design and presentation till date (Moon, 2005). 


\section{History of Physical Model}

Physical models are built to scale. Scaled such that there is a defined ratio between the full size object and its miniature version (Robinson, 2010). Scale modelling has been with us since the beginning of recorded history. They have been used for various reasons throughout history by artists, architects, statesmen, and kings (Neat, 2008). Most importantly being a method of limiting the expenditure of resources prior to an inventor's vision being approved for larger construction by those with power and resources.

Models have not always been a tool for conceptual representation. Its first use, recorded by historians, was for transcendental purposes (Smith, 2004). A medium, employed to shape culture and to control nature. While it earliest recorded document relating to architecture comes from the Roman rule in the 1st century AD.(http:/ / www.modelmaking.co.uk/ a-briefhistory-of-architectural-model-making/ ).In chronological order, the civilization utilization of models in relation to the afterlife is explored, prior to its historical and contemporary use as a tool in the design process.

\subsection{Past}

\subsubsection{Egypt}

An embodiment of the historical Egyptian civilization's belief can be inferred from the function of their pyramids - a machine for eternal life (Smith, 2004). Found in most chambers of the pyramids are intriguing scaled down replica models: a granary, bakery, cattle stable etc. All this served as a necessity for sustenance in the afterlife. Just as the contemporary models are used today to represent what is or could be, the Egyptians models represented the fundamental tenets of their society, as it is used to bolster their lives in the world beyond.

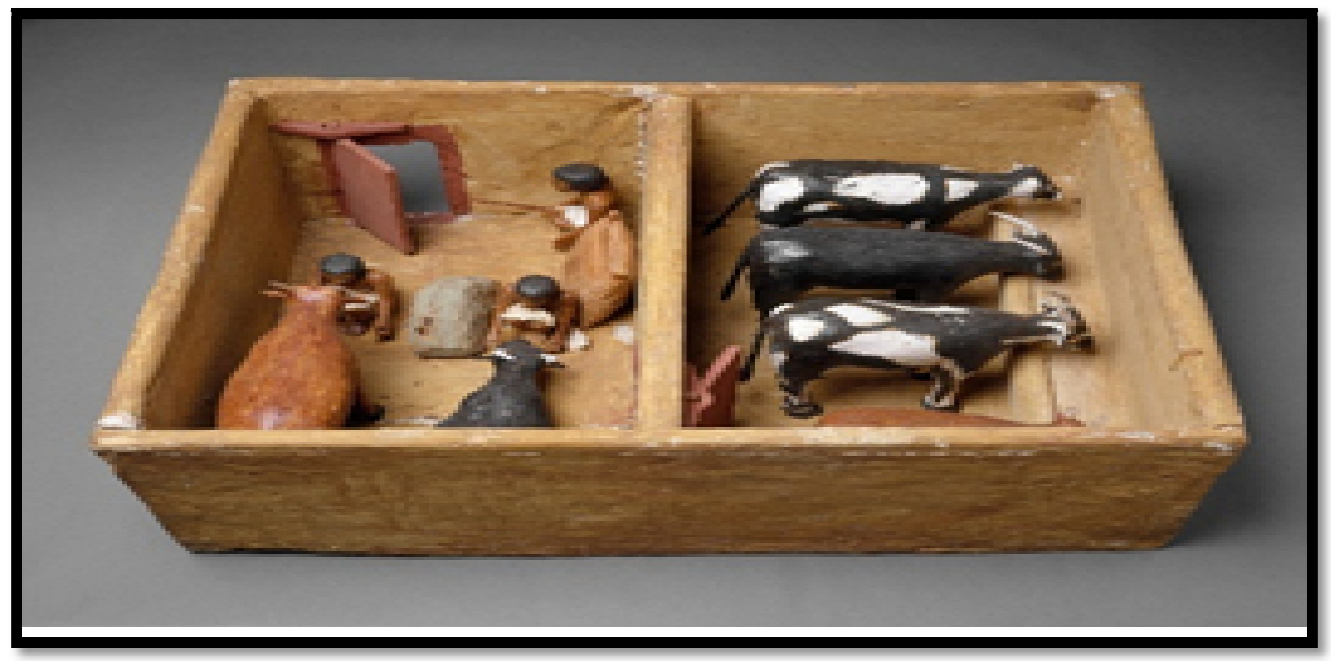

Figure 1: Egyptian Funeral Model: Cattle Stable

Source: The Metropolitan Museum of Art, Accessed February 2018: Www.Metmuseum.Org/ Art/ Collection

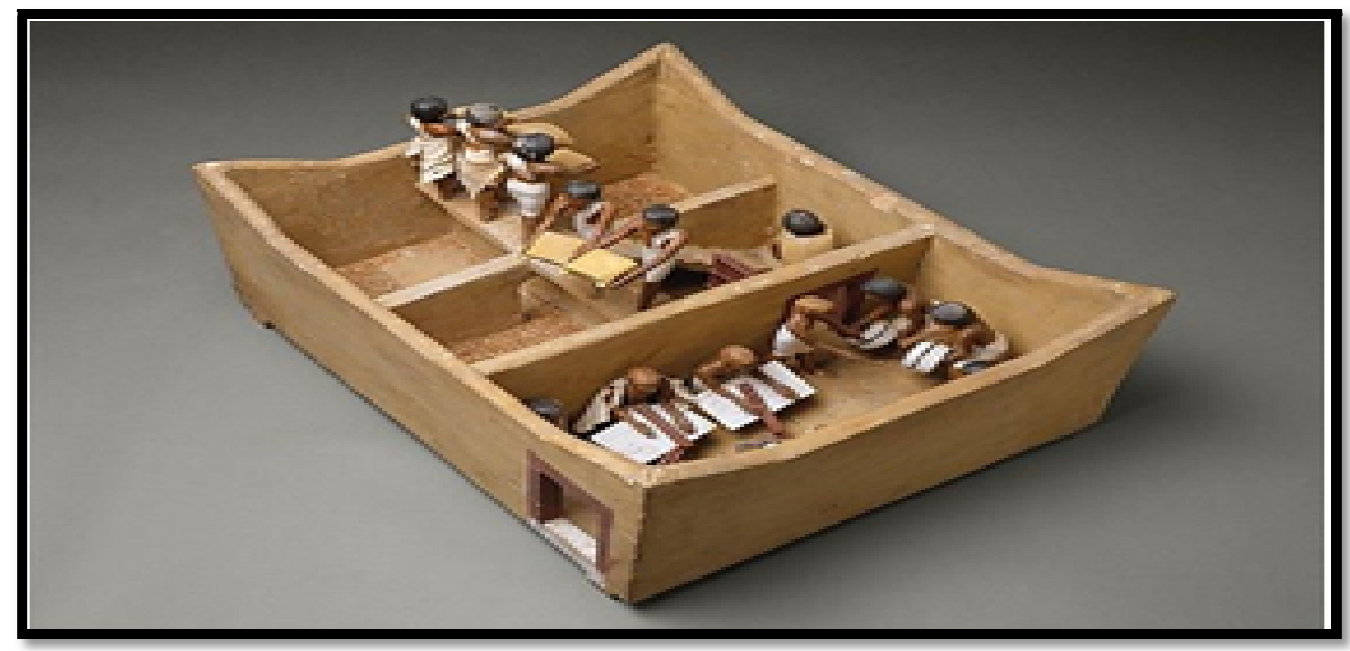

Figure 2: Egyptian Funeral Model: Granary Shop

Source: The Metropolitan Museum of Art, Accessed February 2018: Www.Metmuseum.Org/ Art/ Collection 


\subsubsection{Greek}

In the golden age of Athens', ancient Greece, architecture became the paradigm for western designs. During this period, however, the Greek architects were not held in high esteem compared to the philosophers due to standardization in design (Smith, 2004). With borrowed column designs and plan layout from Egypt and Mycenae accordingly, many important decisions were not made by the architect. He controlled, on the other hand, site work in accordance with prescribed information conveyed to him (Bundgaard, 1957).

With a prevalent design standard, the Greek architect had little use for drawings and models. However, he employed specific one to one models called paradeigma. A paradeigma was used to study specific elements of a total composition such as triglyph, frieze and capital which required a three-dimensional understanding of the designs before their incorporation to the entire system (Coulton, 1977).

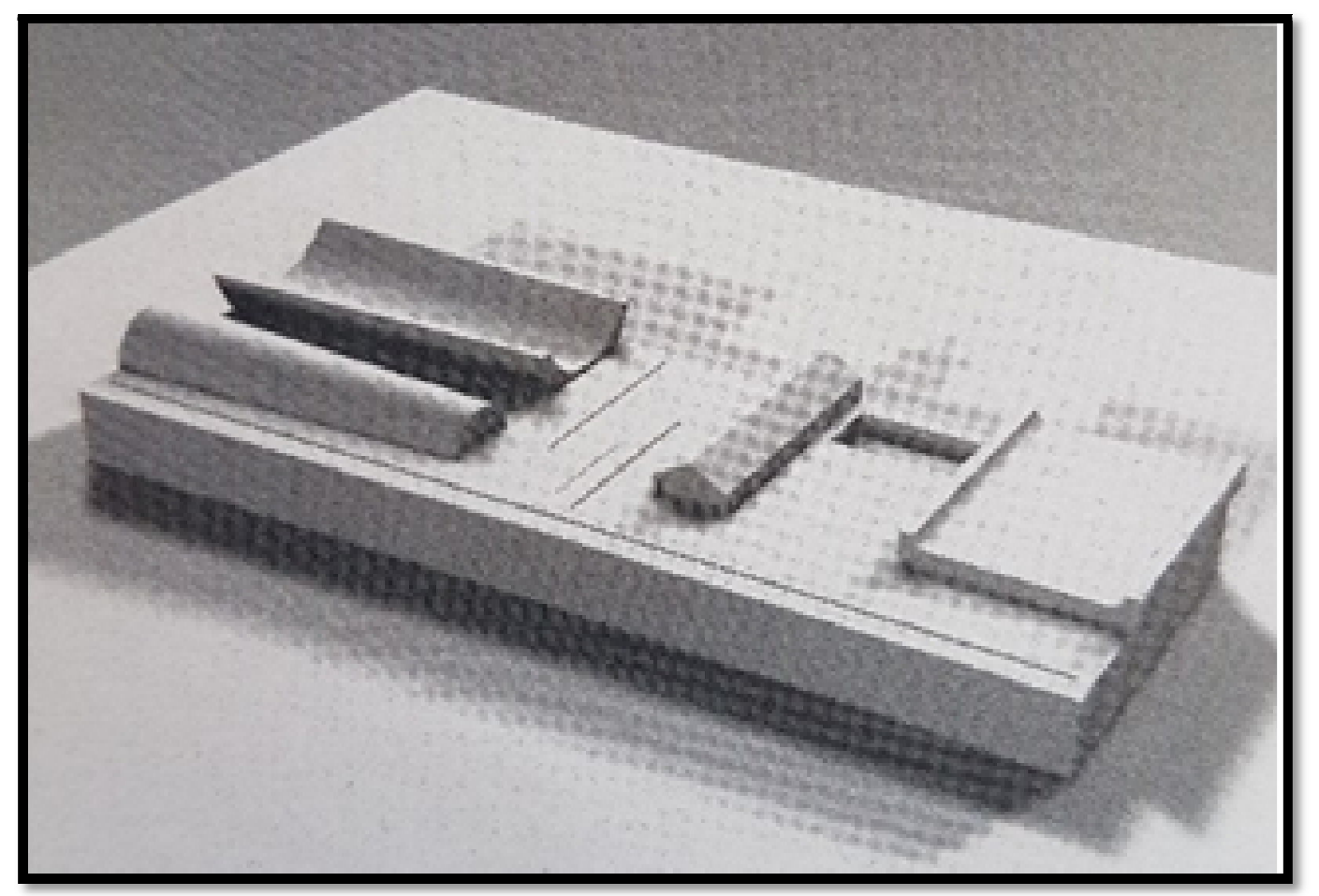

Figure 3: Greek Paradeigma

Source: Albert Smith, Architectural Model As Machine, (Architectural Press, 2004), P.18

\subsubsection{Rome}

The adoption of Greek designs - Doric, Ionic and Corinthian order - in imperial Rome was pushed a step further, surpassing the architecture of the Greeks due to their more advanced technology (Smith, 2004). As a result of the absence of standardization the Roman Architects were responsible for their innovation; thus, their importance in the society. The development of concrete and novel use of bricks made possible the construction of arches, domes and vaults - a great feat in Roman architecture (Smith, 2004).

Extrapolations can be made on early Roman's ability in using the scaled model to propagate ideas to the masses, by looking at insightful stories from Vitruvius' tenth book. He wrote,

"For Diognetus was a rhodian Architect, to whom as an honor, was granted out of the public treasury a fixed annual payment commensurate with the dignity of his art. At this time an Architect from Aradus, Callias by name, coming to Rhodes gave a public lecture, and showed a model of a wall, over which he set a machine on a revolving crane with which he seized a helepolis as it approached the fortifications, and brought it inside the wall. The Rhodians, when they had seen this model, filled with admiration took from Diognetus and transferred this honor to Callias." (Vitruvius\& Granger, 1985)

The passage hints at the scaled model's ability in providing a medium that grants the population an opportunity to perceive a possible future, a similar function to the contemporary architectural model. 


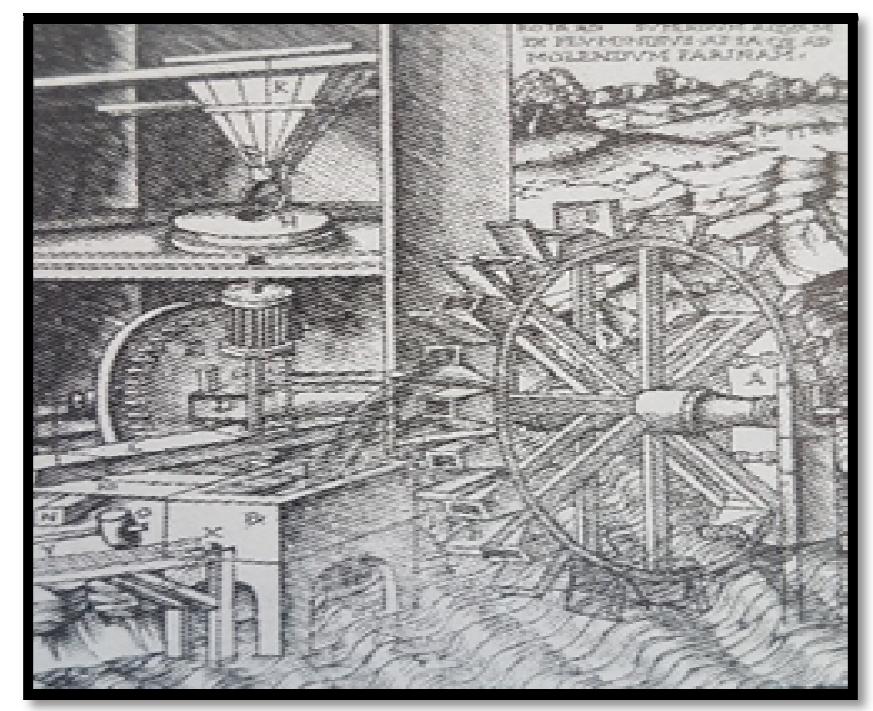

Figure 4: Vitruvian Machine

Source: Marcus Vitruvius Pollio, Frank Granger (Trans) De Architectura,

(Cambridge, MA: Harvard University Press, 1985

\subsubsection{Middle Ages}

The fall of the Roman Empire gave rise to the middle ages. Here, it is difficult to pinpoint the use of small-scaled models in dissipating conceptual thought. However, Albert Smith espouses ideas of the use of scaled one to one models during this era, in his book Architectural Model as Machine(Smith, 2004).His explanation starts with the driving force of this era - Christianity. He notes, accordingly, the influence of the church on European philosophy, science, literature, art and architecture. With a new control in the factors that influences culture, readily, a shift in the architect's definition took place; one who was involved in the design of buildings was termed a 'mechanic'. Becoming a mechanic in the early Middle Ages entails the mastery of a book's curriculum on geometry, based on the doctrine of Heron of Alexandria.

The Architecture of the middle ages can be understood as scaled models because it works in accordance with the function of one. Since the model's main purpose is to propagate an idea, the Gothic churches of the middle ages were scaled models - machines - created by the mechanic to propagate the idea of Christianity.

"...the gothic cathedral is similar to a full-scale model and provides an example of a thinking mechanism used for demonstrating the Christian religion. The building is designed to enhance an awareness of the presence of God and to carefully control the relationship of the parishioners to the church hierarchy. - Albert Smith (Smith, 2004)

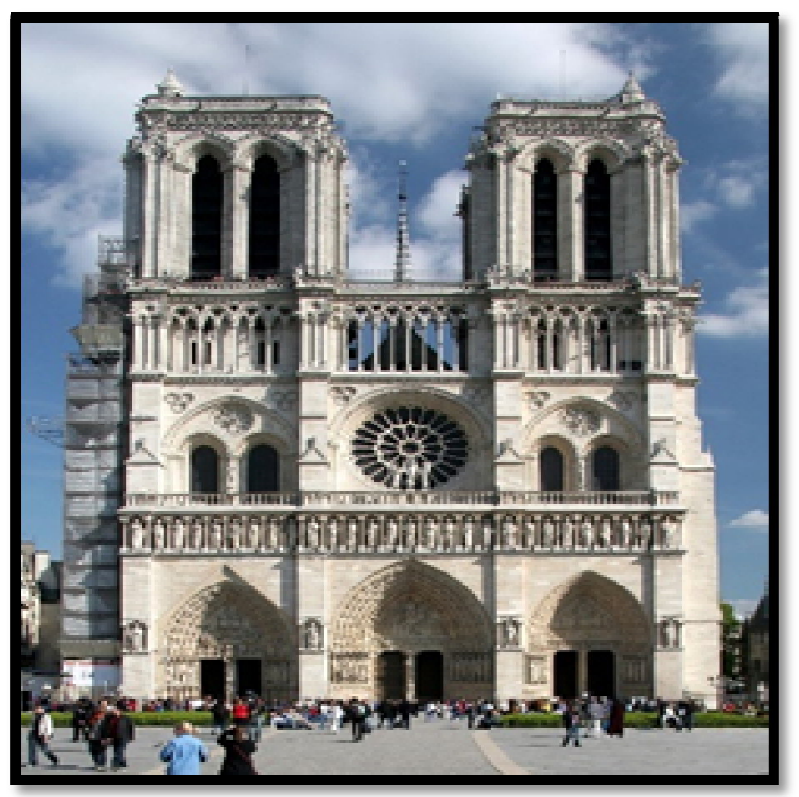

Figure 5: Façade of the cathedral of Notre Dame

Source: ETSU: http:/ / faculty.etsu.edu/ kortumr/ 16highmiddleages/ htmdescriptionpages/ 09notredame.htm 


\subsubsection{Renaissance}

The Renaissance period (1300-1700) marks the use of numerous small-scale model by Italian architects. Very similar to the model's purpose today, they were used in delineating form and technique during the execution of a building. Models made carelessly - lacking detail - were only used to give a general idea of scale and appearance. The lack of details allowed for successive decisions conveyed directly to the builder by the designer (Smith, 2004).

A renaissance man and Italian Architect Leone Battista Alberti (1404-1472) wrote on the importance of the small-scale model in his treatise On the Art of Building in Ten Books. He conveyed that the model had the ability to view the future in more clarity than the drawings allowed, through an anecdote of ancient Rome told by Gaius Suetonius - Julius Caesar had demolished a building because it did not meet his standard (Alberti, 1988).Alberti wrote,

"It (the scale model) will also allow one to increase or decrease the size of those elements freely, to exchange them, and to make new proposals and alterations until everything fits together well and meets with approval... In this way, it is possible to form a clearer and more certain idea of the design and quantity of columns, capitals, bases, cornices, pediments, revetment, flooring, statues, and everything else relating to the construction of the building and its ornamentation."(Alberti, 1988).

It is important to note that he does not see the model as a medium to propagate ideas only, but also as a test bed for new ideas and conceptual designs.

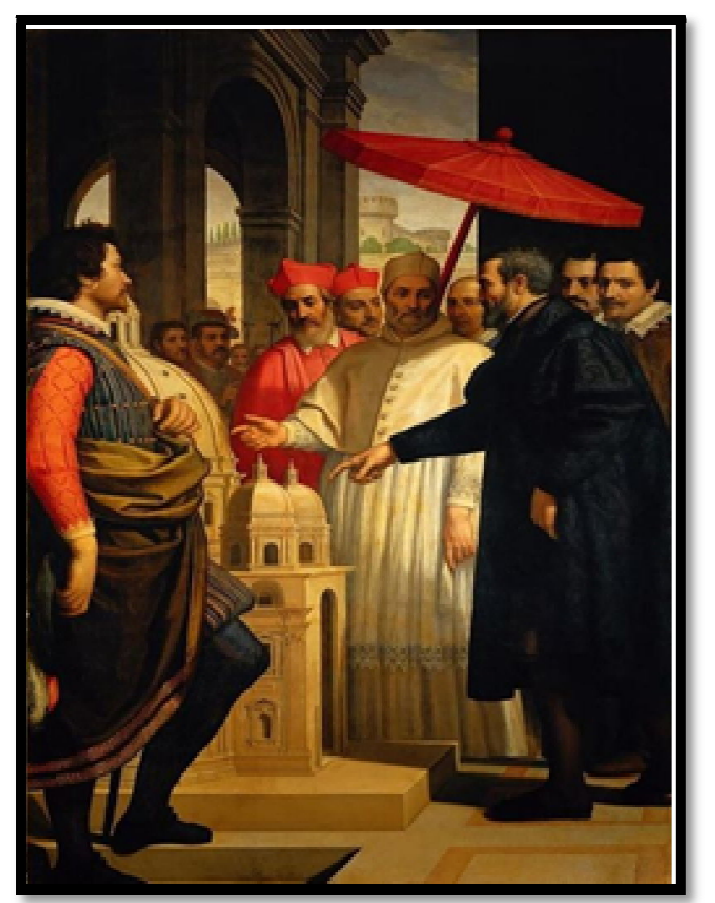

Figure 6 : Painting of Michelangelo Presenting Model to the Pope

Source: Albert Smith, Architectural Model as Machine, (Architectural Press, 2004), P.16.

\subsection{Present}

Today, model styleconsists of a blend of both realism (fully colour, textured and detailed to near real life) and abstraction (idea representation, monochrome usually white or grey). Beyond this is the debate between the options of physical or computer modelling (Phev, 2009).

Computer (Virtual) models have become very popular in recent years due to their efficiency in saving space and time. They enable designers to quickly raise a building out of thin air and add intricate details with a touch of a button. Clients can be taken on a tour inside the proposed building and have a feel of the ambiance. Physical models are often too small to enter but large enough to take up sufficient space in a studio whereas a virtual model resides safely within the computer. It also provides ease in modifications. Buildings can be lengthened, colours changed, trees added, and windows erased in a matter of seconds. Changes to a physical model can take up to hours or even days. However it is important to note that these virtual "threedimensional models" are actually flat, being constructed by a series of two-dimensional images. We live in a three-dimensional world and could best understand a design through physical models.

It may seem like the size of a physical model and the space it takes up will be a drawback compared to a virtual one stowed away in a computer. However, the physical presence of a model actually offers many benefits. It is real and cannot be hidden away with the switch of a button. The architect is forced to look at it even when there is not a desire to do so. It offers thoughtful insight and time to resolve the issues when least expected. Architect Stefan Behnisch says, "Models are always physically visible in the studio, inviting contemplation, comment and change, a process of ripening" (Moon, 2005).

In recent years, architectural model making has undergone a revolution due tothe surge of technological advances that 
makes use of Computer Aided Design (CAD), while still constructing a physical model. The ability to create scale models directly from three-dimensional CAD data in a matter of hours is a reality and the technology is currently being used by key architecture institutions and practices for the creation of proposed and as-built designs.Computer Numerical Control (CNC) milling machines uses $\mathrm{CAD}$ data to quickly cut and produce parts of models.In this process, laser cutting machines are used. What once represented countless hours of hand work with frustrating incisions can now be achieved within minutes and at a level that is near perfection.Using machines to build models offer advantages such as accuracy and extremely fast speeds. These machines can do repetitious tasks, such as punching out thousands of windows, while leaving more time for the builder to invest in creative tasks. Above all, the various parts produced by the CNC machines still have to be assembled by the model maker - the student or the architect.

However, the newest wave in model making is the Rapid Prototyping ( $R P)$. With $\mathrm{RP}$, the human touch is almost completely taken out of the model making process. RP uses CAD data to build the model in layers in just one step. The most popular RP process, known as stereo lithography, uses ultraviolet laser beams to solidify certain parts of liquid polymer resin according to a CAD data. This three-dimensional printers produces mind-bending forms, allowing freedom of expression and a new method of exploring design concepts as they evolve.In the beginning, these machines were only available to large institutions and offices, but with a decline in cost, CNC machines, laser cutters can even be found in small offices (Moon, 2005).

\subsection{Future}

Having machines build models for designers and architects may seem like the perfect solution, but in reality, it can become disastrous. By cutting humans out of the process and relying heavily on machines, we lose the chance for interpretation, modification, and experimentation. The model maker cannot be simply taken out of the equation. It is a dance between the maker and the model, an art of pausing and reassessing that produces unique characteristics and fine craftsmanship (Moon, 2005). The process of makingthe model also helps the maker keep in touch with the real world, for real materials are being put together, rather than just "painting" the ground and walls with an image of grass or stone in the virtual world. Hence, there is a slim chance of constructing a design that would not work in real life if it has already been tested through a physical model, rather than just virtually (Moon, 2005) or through RP.

Virtual models and physical models are two "tool" that should be working together. Each offers its own advantages and makes up for the other's inadequacies. Virtual models are comprised of flat two-dimensional images that cannot offer a complete and coherent view. However, scale models cannot confine the viewer to only the most pleasing view, for it is viewable from all angles. It also cannot give up all the details and specifications in the design.Most interestingly, trend-setting designers and students have freely mixed their tools - virtual as well as physical - which have thereby become truly interactive multimedia.(Jack \& Bram, 2013).By working together, these two types of models gives the most comprehensive presentation of the design. Models will continue to survive as long as designers seek for the best representation of their design. Architect Spencer de Grey believes, "There will always be a demand [for models]. It is difficult to see that fundamentally changing" (Moon, 2005).

It is the best form of representation that can communicate to a large range of people. Peter Pran of NBBJ says "[models have] a kind of universal language. Everybody can understand a model ...they [model] speak to us all" (Moon, 2005). It requires no background training for architectural drawings, computer familiarity for virtual fly-bys, or the imagination to stitch a series of perspective drawings together. The scale model is the complete realization of the design, sitting quietly in front of the viewer and designer to explore and understand.

Models will continue to grow and evolve with the changes in materials and technology, but the purpose of the threedimensional model will not been altered. It will always stand as a design tool and communication device. Although fewer are being built today, models are vital tools that can never be completely replaced. They allow designers to visualize relationships between the different elements that would be impossible to do on a computer (Moon, 2005). Most professional studios will make use of models in their design and presentation. Some will have an outside source perform the more complicated tasks, such as laser cutting for precise contour landmasses, but will build the rest in-house (Yan, 2009). Having skills in model making will give you an advantage in conveying your ideas over a competitor who has no experience using this valuable tool.

\section{The Design Process}

Architecture being an art, makes designers and scholars of architecture resort to visual modes of communication (Jack $\& B r a m, 2013)$, which includes; drawings, paintings and other graphic renderings for presentation. Aside these, the only other leading medium' is undoubtedly the architectural Model, which can bemore appealing than the drawing. (Jack \&Bram, 2013).

Models have a tangibility that other presentation methods do not have; their multiple vantage points enable the discovery of unforeseen conditions that one-directional drawings may overlook; and their abstraction focuses attention on qualities of space and scale. (Phev, 2009).

As a part of the design process, models provide a fairly quick means of exploring different things, from the form of the overall building to a detail where two materials meet. Often times, a quick model (working model) will reveal possibilities that cannot be seen from sketching or drawing.Virtualmodels may be more precise, but it may not yield the same discoveries that physical models allow. For instance, during the planning and design stage, a physical model is used in the first conceptual expansion phase, and then virtual rendering (VR) is applied in the convergent design phase (Koga et al., 2008 as cited in Lei et al., 2014).However, virtual environments (VEs) are seldom used in actual creation, form-finding, and collaboration in 
architecture. (Lei et al., 2014). In effect, the outcome of an experiment performed (Differences in Spatial Understanding Observed by Using Physical and Virtual Models) shows that the physical model tends to allow quicker and more accurate comparison of building heights compared with the virtual model. Therefore, a physical model is more intuitive than a virtual model as a spatial understanding model. (Lei et al., 2014).

Today, the increasing ease with which computer technology can be utilised results in students avoiding the use of physical models. Instead they tend to favour the development of three-dimensional computer models. Before the advent of computers, lecturers do not encourage this practice and they believe that physical models still allow the best exploration within the design process. (Hermie \&Jolanda, 2010).

This idea of the importance of model making as a design tool was questioned by S. Hohauser (1970) in this manner; "why build physical models when sketches can serve as adequate presentations?" "Why design a three-dimensional object in a two-dimensional study medium?" According to Hohauser, it is more effective for designers to tackle three-dimensional problems with a three-dimensional form. Hence, model making should be a critical aspect of the design process and not supposed to be relegated to the background. This must have informed the statement by Karen Moon, that "Designing by model making lets you face the problem directly and embrace the spontaneous and serendipitous" (Moon, 2005, p. 51).The use of model has a design tool is exemplified by one of the greatest architects of our time, Frank Gehry.In the documentary; "Sketches of Frank Gehry", whereinhe is seen to be designing and had resolved design issues for all his projects with the use of models (working models). Both in his Master Class and in practice.

Unfortunately, the sequence we see today in the design process among students in schools of architecture and most architects in practice is; sketches first, drafting follows, computer renderings and physical models often requested last for presentations. What if the sequence were reversed? Where physical models are used as "design tools" in the design process and not just meant for mere presentation. How would this impact on the ability to conceptualize and visualize? And what consequence does this have on the design outcome?

\section{The Architectural Design Process}

According to Mark D. Gross (Gross \& Do, 1997:4), "the lack of formal methods in architectural design puzzles each generation of students entering studio; they learn the how to' skills through imitation of their teachers and more senior classmates. A clear indicator that many universities have no formal methodological approach to architectural studio teaching.

As a studio culture, it is expected that each student must independently develop his/ her own process and method of design. This rarely comes from the teachers. Most studio critic are familiar with studio situations, but not necessarily with the theory that under pins design-studio teaching and learning. Often the value of the design process and the development of a strong architectural idea are not taught.

However, recent research and literature on teaching indicate that many educators are investigating a more formal instructional method of design teaching and learning in the studio and are seeking to understand the theory behind the learning that takes place. (Hermie \& Jolanda, 2010). This suggests that the lecturer in the studio "has to introduce many different types of didactics to build up students' design capacity" (Jiun-De \& Heylighen, 2006).

Going by this, the Cape Peninsula University of Technology (CPUT), South Africa, carried out a study on the pedagogical studioteaching approach in the undergraduate design studio, where making of the working models was the major part of the studio activity, and reported by (Hermie \& Jolanda, 2010). Two case studies were presented to illustrate the importance of the use of physical models. The process started with a simple site model, from which a first architectural idea was developed. The models varied in scale and detail, but all contributed to the development of an appropriate and integrated response to the design problem. They helped the student store cognise and develop their main architectural idea from concept to detail. They served as physical evidence of a student's thought process and development. Unexpected and unintentional ideas often developed from these models.

Active reflection within the process was encouraged. As Barbaradela Harpe (Harpe et al., 2009) observed: "student learning was deepened and shifted from a focus on the final design artefact or product to the process of learning, through a cycle of continuous reflection". The focus is thus on the process rather than on the product. It was based on this that Kaufman and Glaser wrote about the value of an idea that: "in its simplest form the idea is the basic building block of solution, creativity and innovation" (Kaufman \& Glaser, 2004). They further argue for ideas to be concretely represented for them to be of the greatest value: "The experience of the writers has led to the belief that ideas in the mind are nothing, an idea that is spoken is transient, an idea that is written is fixed, and an idea that is visualized is salient", and through their investigation conclude that "...students responded well to learning a design process that focuses on idea generation" and "...data shows that students build trust and confidence in their own intuition allowing them the ability to generate many ideas" (Kaufman \& Glaser,2004).

\section{Model as Design Tool}

Although the study described above took place in a structured design studio, there are still possibilities for enhancing the learning process for the students. Ellmers and Foley specifically refer to "further developing of learning activities specifically engaging with reflective practice" (Ellmers \& Foley, 2007) within an already structured environment. 
Physical models as a vehicle for process, idea and reflection seemed the appropriate choice in the study. The working model as a specific graphic communication medium was used as a summary of ideas, define a specific place in the process and provide a physical form for reflection. Webster (2001) used the Design Diary as such a specific vehicle in the studio. It is described as a "container" and as a "central location for both recording and reflecting on the process of design" (Webster, 2001) and it was found that it also helped to "enhance the effectiveness of the reflective stage in the learning cycle" (Webster, 2001). Webster also referred to a specific practical application: "unlike the normal working method, where students continuously use drawings to help the ideation phase, and as a result amass piles of doodles and sketches which mean little to them after the event and usually mean nothing to an external observer, the Design Diary 'working method' encourages students to reflect on their personal doodles and to transpose them into a form which explicitly communicates their ideas and explorations". The physical act of making something is also described as very valuable by Philippou who wrote that "the craft of making functioned as a vehicle for thinking ideas in concrete matter" (Philippou, 2001).

The cognitive focus of the physical model is that it enhances dialogue. Not only does the model talk back, but it is an easy graphic form to access visually and verbally for both student and lecturer. This is in contrast to the accessibility of three-dimensional computer drawings. These are often only accessible within a "live" computer where students can show the whole model with ease. However, students tend to print out views that hide problematic issues - this is not possible to achieve with a physical model. It is important to note that the models described here are working and not presentation models. They can be made cheaply from left-over materials.

Hence, the appropriateness of the working model as a design tool.

\section{Conclusion}

Form the study carried out at CPUT, it is evident that working models can be made cheaply from left-over materials and this has made models less "precious" and altered the perception that once something is relatively "concrete", it cannot be improved or changed. Sarah Kuhn (2001) emphasises the value of early commitment to an idea and also the "rapid proliferation of potential design solutions". She also wrote that "... a three dimensional model of the building, or a visit to the site, may offer a fresh way of seeing the problem and of approaching solution" (Kuhn, 2001). The models indeed provided opportunities for experimenting and taking apart ideas. The models help to control the process, make it clearer and more transparent. They also help to force active participation. In addition to idea development, models help to physically retain the design idea in conceptual development. And compared to other graphic forms, the model is the best three-dimensional communication about the initial idea. It is easily accessible and easy to refer back to. It is possible to look at various possibilities within the main idea.

Rather than relegating models making to the last in the design process - as models open up a realm of creativity and freethinking, allowing the maker (students and architects alike) to experiment with the boundaries of the unbuildable. There exists a strong connection between models and childhood playtime, where there lies elements of fun and freedom, creativity and spontaneity. Like children playing with dollhouses, architecture students and architects in practice making models have the chance to experiment with their imagination and come up with new expressions. - they should occur at every stage in the design process to inform decisions for the best outcome.

\section{References}

i. Salama, A. \& Wilkinson, N. (2007). Design Studio Pedagogy: Horizons for the Future. Gateshead, U.K.: The Urban International Press.

ii. Robinson, L. (2010). A Brief History of Modern Miniatures. A Publication to Benefit the San Lorenzo Valley Museum.

iii. Phev, K. (2009). A Guide to Model Making for the Landscape Architecture Student. University of California - Davis. June $12,2009$.

iv. Moon, K. (2005). Modelling Messages. New York: Monacelli. p.11-51, 205-208.

v. Neat D. (2008). Model-Making. Swindon: Crowood Press.

vi. Lei, S., Tomohiro F., Toshiki T., \& Nobuyoshi Y. (2014). Differences in Spatial Understanding between Physical and Virtual Models. Frontiers of Architectural Research. Volume 3, Issue 1, March 2014, Pages 28-35.

vii. Hermie, D.V., \& Jolanda V. M. (2010). The Importance of Physically Built Working Models in Design Teaching of Undergraduate Architectural Students. Connected 2010 - 2nd International Conference on Design Education 28th June-1st July 2010, University of New South Wales, Sydney, Australia.

viii. Jack, B. \& Bram, B. (2013). The Model as the Method. Precedent-based architectural design exploration and communication. Form \& Modelling Studies Faculty of Architecture Delft University of Technology Delft, Netherlands.

ix. Dela Harpe, B., Peterson, J.F., Frankham, N., Zehner, R., Neale, D., Musgrave, E., \& McDermott, R. (2009). Assessment focus in studio: what is most prominent in architecture, art and design? International Journal of Art and Design Education, 28(1):37-51, March 20. 
x. Ellmers, G. \& Foley, M. (2007). Introducing reflective strategies informed by problem-based learning to enhance cognitive participation and knowledge transference in graphic design education. Proceedings of 2007 Connected. 28th June - 1st July 2007.

xi. Gross, M. D. \& Do, E.Y. (1997). The design studio approach: Learning design in architecture education. In Design Education Workshop, J. Kolodner \& M. Guzdial (eds). Georgia Institute of Technology, 8th Sept - 9th Sept.

xii. Hohauser, S. (1970). Architectural and Interior Models - Design and Construction, Van Nostrand Reinhold Company, New York.

xiii. Jiun-De, C. \& Heylighen, A. (2006). Learning Design Teaching. In Al-Qawasmi, J \& Vasquezde Velasco, GP (eds). 2006. Changing Trends in Architectural Design Education. Morocco: CSAAR. p.557-587.

xiv. Kaufman, J. \& Glaser, M. (2004). A product design studio for non-designers: Teaching innovation to non-designers. Proceedings of the 2004 Eastman National IDSA Education Conference, Pasadena, 24th Oct. - 26th Oct. 2004, Art Centre College of Design.

xv. Kuhn, S. (2001). Learning form the architecture studio: Implications for project based pedagogy. International Journal of Engineering Education, 17 ( 4 \& 5): 349-352, 2001.

xvi. Werner, M. (2010). Architectural Model Building. Princeton Archit.Press, 2010, p.11.

xvii. Smith, A. (2004). Architectural Model as Machine. Architectural press, 2004.p.4 - 25

xviii. Bundgaard, J.A., Mnesicles. (1957). A Greek Architects at work, Copenhagen: Scandanavian University Press, 1957.

xix. Coulton, J.J. (1977). Ancient Greek Architects at Work. Ithaca, NY: Cornell University Press, 1977. p.1-2.

xx. Vitruvius, M. P., \& Granger, F. (1985). (Trans) De Architectural. Cambridge, MA: Harvard University Press, 1985. p.7-11. xxi. Alberti, L.B. (1988). On the Art of Building in Ten Books. Cambridge, MA: The Mit Press, 1988. p.33, 34.

xxii. A Brief History of Architectural Model Making. Retrieved from; http://www.modelmaking.co.uk/ a-brief-history-ofarchitectural-model-making/

xxiii. Scale Models through the Ages. Retrieved from; https://www.toysperiod.com/blog/scale-models/scale-modelsthrough-the-ages/

xxiv. Philippou, S. (2001). On a paradox in design studio teaching or the centrality of the periphery. Proceedings of 2001 AEE 2001. 11 Sept-12 Sept 2001. Cardiff. Retrieved 10 Jan 2010 from http:/ / www .cebe.heacademy.ac.uk/ aee/ papers.html.

xxv. Webster, H. (2001). The Design Diary: Promoting reflective practice in the design studio. Proceedings of 2001 AEE 2001. 11 Sept-12 Sept 2001. Cardiff. Retrieved 10 Jan 2010 from http:// www .cebe.heacademy.ac.uk/ aee/ papers.html.

xxvi. The Architects' Take: News and Discussion from an Architect's Viewpoint. Entry: Why We Still Build Models. Tuesday, January 27, 2015 | Mark English, AIA | Mark English Architects, Work/ News. https:// thearchitectstake.com/ worknews/ why-we-still-build-models/ Accessed February, 2018.

xxvii. Sketches of Frank Gehry 2006, Sydney Pallock, Los Angeles. 